\title{
Effect of Inclination and of Darcy Number on Bifurcations and Thermal Transfer in a Square Porous Cavity
}

\author{
Sabiha Aklouche-Benouaguef ${ }^{1}$, Saad Adjal ${ }^{1}$, and Belkacem. Zeghmati ${ }^{2}$ \\ ${ }^{1}$ Laboratory of Polyphasic Transport and in Porous Media.LTPMP. USTHB.Algerie. \\ ${ }^{2}$ Laboratory of Mathematical and of Physical System, Group of Mechanics LAMPS, Via Domitia, Perpignan
}

\begin{abstract}
In the present work a numerical study of natural convection in air in a square cavity filled with a porous media, was carried out in order to analyze the effects of the inclination angle and the Darcy number on the roads toward the chaos. The horizontal wall of the cavity is adiabatic while the vertical walls, composed of two part of the same size, are maintained at different temperatures. The dimensionless transfer equations, expressed in terms of stream function and vorticity had been solved using the Alternating Direction Implicit Method (ADI) and the GAUSS elimination method. The different bifurcations had been represented by attractor in phase space and by amplitude spectrum. We have noticed that for an inclination angle equal to $30^{\circ}$, the scenario of the roads towards the chaos is in agreement with the one of Feigen Baum. For the inclination angle equal to $60^{\circ}$, the scenario is that of Ruelle and Takens which is by quasi periodicity.
\end{abstract}

\section{Nomenclature}

a Thermal diffusivity of the fluid $\left(\mathrm{m}^{2} \mathrm{~s}^{-1}\right)$

$\mathrm{Cp} \quad$ Fluid Thermal Capacity $\left(\mathrm{j} \cdot \mathrm{Kg}^{-1} \cdot \mathrm{K}^{-1}\right)$

Da Number of Darcy

Gr Number of Grashof

$\mathrm{H} \quad$ Height of the cavity $(\mathrm{m})$

$\mathrm{K}_{\mathrm{f}} \quad$ Thermal conductivity of fluid

$\mathrm{K}_{\mathrm{s}} \quad$ Thermal conductivity of solid

$\mathrm{K}_{\text {eff }} \quad$ Effective thermal conductivity

$\mathrm{Nx} \quad$ Number of nodes following ox

Ny Number of nodes following oy

$\mathrm{R}_{\mathrm{v}} \quad$ Viscosity ratio $\mathrm{R}_{\mathrm{v}}=\mu_{\mathrm{eff}} / \mu_{\mathrm{f}}$

$\mathrm{R}_{\mathrm{k}} \quad$ Conductivity Ratio $\mathrm{R}_{\mathrm{k}}=\mathrm{k}_{\mathrm{eff}} / \mathrm{k}_{\mathrm{f}}$

$\mathrm{T}_{\mathrm{C}} \quad$ Cold temperature

$\mathrm{T}_{\mathrm{h}} \quad$ Hot temperature $\sigma=(\rho . c p)_{\mathrm{eff}} /(\rho . c p)_{\mathrm{f}}$

\section{Introduction}

Natural convection in porous media were the subject of several studies, due to the large number of the industrial applications such as: geothermal reservoir flow, separation processes in the chemical industries, dispersion of chemical contaminants through waterlogged soil, moisture in water systems, grain storage, oil production etc. A set of applications about convective flows in porous media had been studied by Kaviany [1] and Nield and Bejan [2]. This includes vertical and horizontal porous beds which are part of the work of Poulikakos and Bejan [3]. Asymmetric oscillations had been observed by Mamou et al [4] in a horizontal layer of fluid in thermo-solutal convection. Results presented show that for a diffusivity of the solute smaller than the thermal diffusivity, a thermal instability develops, characterized by a bifurcation of HOPF. Merrikh et al [5] simulated numerically the natural convection in a cavity filled with two layers of medium porous.

The main aim of this study was the validation of the Darcy model for different combinations of the Darcy number, the Rayleigh number and the permeability. It was been shown that the flow structure and the heat transfer can be different of those predict by the Darcy model. Basak et al. [6] have numerically studied the natural convection in a square cavity filled with a porous medium. Transfers in the porous medium are described by the Darcy-Forchheimer model. The calculations had been performed for Darcy and Prandtl numbers varying respectively between $10^{-5}-10^{-3}$ and between $0.71-10$.

At our knowledge there is very little works available on the chaotic transient characteristics of natural thermal convection in a porous cavity. The effect of wave phenomena on chaotic convection in porous medium employing Darcy model had been investigated by Vadász et al. [7]. Their results showed that periodic solutions and chaotic solutions alternated as the Rayleigh number varies. A continuation of the works of Vadasz et al. was carried out by Kiran et al. [8] in order to analyze the chaos phenomenon in a saturated porous layer of bottom-heated fluid and subjected to temporal temperature modulation. Bhadauria et al. [9] had investigated chaotic and oscillatory thermal instability in a viscoelastic magnetic fluid layer. They found that oscillatory convection enhances heat and mass transfer in the system in comparison with stationary case. In

* Corresponding author Email: mesadz@yahoo.fr 
addition they justified that gravity modulation can be used to alter the chaotic nature of the phenomenon. Vadasz [10] was interested by the control of the transition from the convective fixed point to the chaos in rectangular fluid-saturated porous domain heated. He presented and compared three methods to solve the equations of the natural convection: Analytic (weak nonlinear), calculation (adomian decomposition) as well as numerical (Runge-Kutta-Verner). The solution of the point of transition to chaos deduced from the analytical method is identical to the one obtained using numerical procedures in the vicinity of a convective fixed point. The described literature shows that a cavity filled with porous medium in which the horizontal walls are adiabatic while the upper half-vertical walls are maintained to a cold temperature and the lower halfvertical walls are hot has not been studied. The aim of the present study is to analyse the effects of the cavity inclination angle and the Darcy number on the heat transfer, the roads towards chaos and the different type of attractors.

\section{Analysis and modelling}

Figure 1 represents the schematic physical model. It consists of a square cavity filled with a porous medium. The horizontal walls are adiabatic while the vertical walls are heated in the lower half and cooled in the upper half. This cavity is inclined respect to horizontal of an angle $\alpha$. Thermal transfer is governed by the equations of natural convection in a porous medium. We adopt the following hypotheses: The physical properties of the fluid are constant excepted the density variation which induces the forces of gravity and which obeys the Boussinesq approximation. Viscous dissipation is negligible in the equation of energy. The flow is laminar. The fluid is incompressible. The radiation transfer is negligible. The air is assimilated to a perfect gas. The porous medium and the fluid are in local thermal equilibrium.

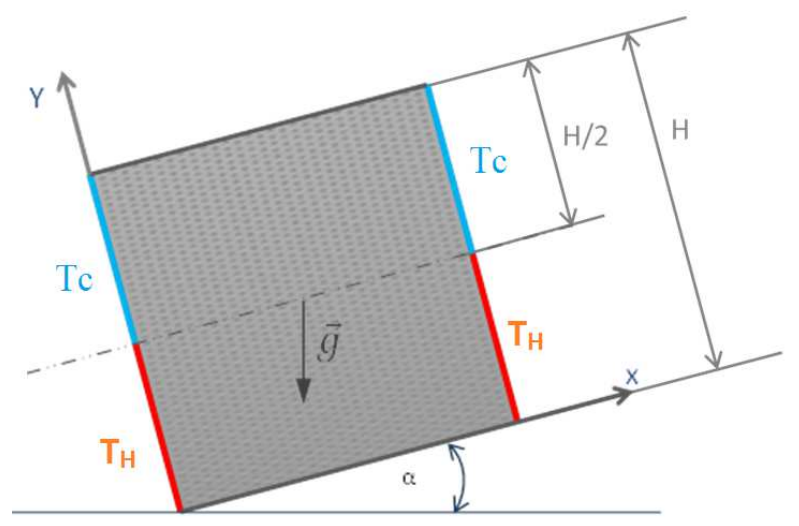

Fig. 1. Physical Configuration.

Taking account of the above assumptions transfer equation, the governing equations and the boundary condition are cast in dimensionless form using the dimensionless variables:

$$
\begin{gathered}
\mathrm{x}=\frac{\mathrm{x} *}{\mathrm{H}}, \mathrm{y}=\frac{\mathrm{y} *}{\mathrm{H}}, \mathrm{u}=\frac{\mathrm{u} \cdot \mathrm{H}}{\mathrm{a}}, \mathrm{v}=\frac{\mathrm{v}^{*} \cdot \mathrm{H}}{\mathrm{a}} \\
\mathrm{t}=\frac{\mathrm{t} * \mathrm{a}}{\mathrm{H}^{2}}, \quad \mathrm{~T}=\frac{(\mathrm{T} * \text {-Tcold })}{(\text { Thot }- \text { Tcold })} \\
\Omega=\frac{\Omega^{*} \cdot \mathrm{H}^{2}}{\mathrm{a}}, \quad \Psi=\frac{\Psi^{*}}{\mathrm{a}}, \mathrm{Da}=\frac{\mathrm{K}}{\mathrm{H}^{2}} \\
\mathrm{R}_{\mathrm{k}}=\frac{\mathrm{K}_{\text {eff }}}{\mathrm{K}}, \quad \mathrm{R}_{\mathrm{V}}=\frac{\mu_{\text {eff }}}{\mu}
\end{gathered}
$$

The resulting non-dimensional equations are: Continuity equation.

$$
\Omega=-\frac{\partial^{2} \Psi}{\partial x^{2}}-\frac{\partial^{2} \Psi}{\partial y^{2}}
$$

Vorticity equation

$$
\begin{gathered}
\varepsilon \frac{\mathrm{d} \Omega^{*}}{\mathrm{dt} *}+\frac{\partial}{\partial \mathrm{x} *}\left[\left(\mathrm{u} * \Omega^{*}\right)-\varepsilon^{2} \mathrm{R} v v \cdot \mathrm{P}\left(\frac{\partial \Omega^{*}}{\partial \mathrm{x} *}\right)\right]+ \\
\frac{\partial}{\partial \mathrm{y} *}\left[\left(\mathrm{v}^{*} \Omega^{*}\right)-\varepsilon^{2} \mathrm{R} v v \cdot \mathrm{P}\left(\frac{\partial \Omega^{*}}{\partial \mathrm{y} *}\right)\right]= \\
\varepsilon^{2} \operatorname{RaPr}\left[\cos \alpha\left(\frac{\partial \mathrm{T} *}{\partial \mathrm{x}^{*}}\right)-\sin \alpha\left(\frac{\partial \mathrm{T} *}{\partial \mathrm{x}^{*}}\right)\right]-\varepsilon^{2} \frac{\mathrm{Pr}}{\mathrm{Da}} \Omega^{*}
\end{gathered}
$$

Energy equation

$$
\sigma \frac{\partial T}{\partial t}+\frac{\partial}{\partial x}\left(u T-R_{k} \frac{\partial T}{\partial x}\right)+\frac{\partial}{\partial y}\left(v T-R_{k} \frac{\partial T}{\partial y}\right)=0
$$

In addition the initials and boundary conditions are take the following form :

-Initials conditions

$\mathrm{t} \leq \mathrm{t}_{0}$, to, being the dimensionless time from which the half parts of the vertical walls are subjected to temperature Tc and Th.

$$
\begin{aligned}
& 0 \leq \mathrm{x} \leq 1,0 \leq \mathrm{y} \leq 1 \\
& \mathrm{~T}=0 \quad \Omega=0 \quad \Psi=0 \quad \mathrm{u}=0 \quad \mathrm{v}=0
\end{aligned}
$$

-Boundary conditions

$t \geq t_{0}$

$$
\begin{aligned}
& x=0, x=1, o \leq y \leq 1: \\
& u=0 v=0 \quad \Psi=0 \\
& x=0, x=1,0 \leq y \leq 1 / 2, T=1
\end{aligned}
$$

$$
\begin{aligned}
& 1 / 2<y \leq 1, T=0 \\
& \mathrm{~T}\left(\mathrm{x}, \frac{\mathrm{H}}{2}\right)=0.5 \\
& 0 \leq x \leq 1, y=0, y=1:
\end{aligned}
$$

$$
u=0, v=0, \Psi=0,\left.\frac{\partial T}{\partial y}\right|_{y=0,1}
$$


The equations (5-7) associated on initial and boundary conditions (8-11) are solved using the implicit method in alternate directions (A.D.I), the GAUSS elimination method and an iterative procedure. The velocity field is deduced by using cantered differences of order two. At each time step, the solution is deemed converged when the convergence criterion for the stream function is less than $10^{-5}$. For, the temperature and vorticity the convergence criterion is falling under $10^{-6}$ (Equation 12).

$$
\max _{p=1} \frac{\sum_{i} \sum_{j}\left|f_{i j}^{p, n+1}-f_{i j}^{p, n}\right|}{\sum_{i} \sum_{j}\left|f_{i j}^{p, n+1}\right|} \leq 10^{-6}
$$

Global Nusselt number along the hot part vertical wall

$$
N_{u}^{H o t}=\int_{0}^{1 / 2}-\left(\frac{\partial T^{*}}{\partial x^{*}}\right)_{x=0} d y+\int_{0}^{1 / 2}-\left(\frac{\partial T^{*}}{\partial x^{*}}\right)_{x=1} d y
$$

Global Nusselt number along the cold part vertical wall

$$
N_{u}^{\text {Cold }}=\int_{1 / 2}^{1}+\left.\left(\frac{\partial T^{*}}{\partial x^{*}}\right)\right|_{x=0} d y+\int_{1 / 2}^{1}-\left.\left(\frac{\partial T^{*}}{\partial x^{*}}\right)\right|_{x=1} d y
$$

\section{Results and discussion}

In order to validate our approach, we applied our numerical code to the De Vahl Davis problem [11]. (Figure 2), and to the one of Ndame [12]. (Figure3). As is can be seen in figure 3 our result concerning the temperature evolution versus the abscissa is in good agreement with the one of Ndame. Computations have been performed for two inclinations (30 and 60 degrees) and two Darcy number $\mathrm{Da}=10^{-3}$ and $\mathrm{Da}=10^{-3}$. The discrepancies are about $0.6 \%$. For the temperature. The value found indicates the validity of our calculation code.

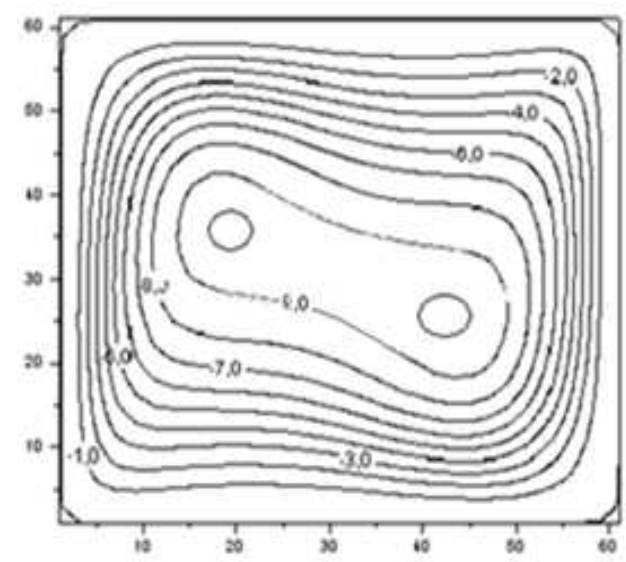

Fig. 2. Physical Validation with De Vahl Davis (Stream function, our work) For $\mathrm{Ra}=10^{6}$

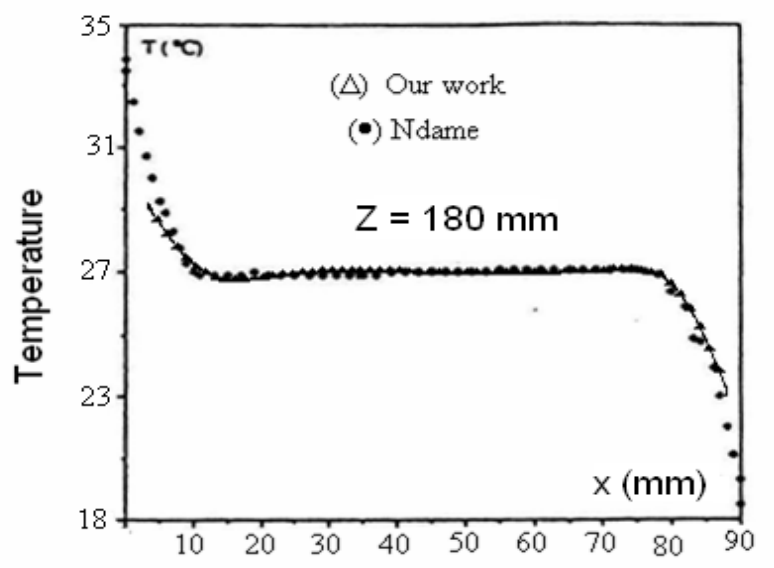

Fig. 3. Temperature evolution versus the abscissa

We analyze the effect of the inclination angle $\left(30^{\circ}\right.$ and $60^{\circ}$ ) of the cavity on the scenario towards chaos for a porosity of the porous medium equal to 0.45 and for two Darcy numbers $\mathrm{Da}=10^{-3}$ and $\mathrm{Da}=10^{-5}$. The roads to chaos borrowed by the system are represented by attractors in phase spaces. The stationary solution is evidenced by a spiral ending in a limit point, while the limit cycle characterizes the unsteady solution. For the inclined cavity $(\alpha=30$ and $\mathrm{Da}=10^{-3}$ ) the limit point attractor is obtained for a Rayleigh number equal to $3.9 \times 10^{-5}$ (Figure. 4 a). The figure $4 \mathrm{~b}$ represents the time evolution of a parameter dynamic which is the number of Nusselt. There is a damping of the oscillations which show the stationary of the system for an infinite time. The first bifurcation of HOPF characterized by the appearance of the limit cycle corresponds to a critical Rayleigh number $\mathrm{Ra}=5 \times 10^{5}$, a time step equal to $9 \times 10^{-6}$ and a mesh size $101 \times 101$. The amplitude spectrum of the oscillatory solution is highlighted in Figure 5. We noticed that the results become sensitive to space steps and time steps as the Rayleigh number increases. The frequencies of the unsteady solution are obtained by using the fast Fourier transform with a spectral resolution less than 0.2. We note that the amplitudes are less sensitive than the frequencies to space steps and time steps. An instability appears as a cascade with harmonics (Figure 6) characterizing the quasi periodicity of the system, and another instability with under harmonics (Figure 7) which is a doubling period. These two bifurcations appear respectively for a Rayleigh number $\mathrm{Ra}=8 \times 10^{5}$, and $\mathrm{Ra}=1.5 \times 10^{6}$. The figures show that the energy dissipated in the system is very important given the peak values. This doubling of period is in agreement with the Floquet theory [13]. Chaos settles for an intensification of natural convection corresponding to $\mathrm{Ra}=3 \times 10^{6}$ 


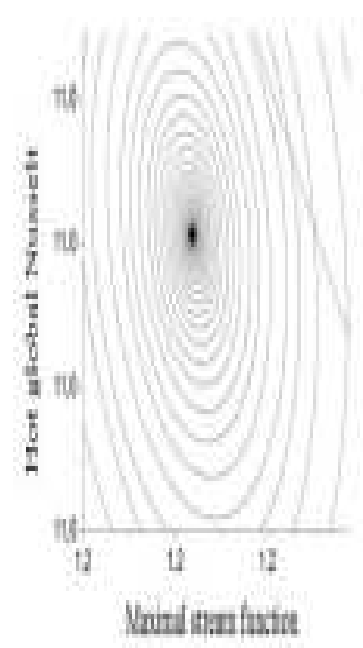

(1)

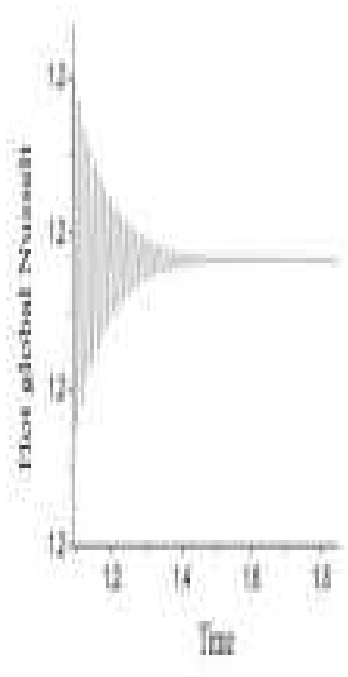

(b)
Fig. 4. a) Limit Point Attractor b) Temporal Evolution $\mathrm{Da}=10^{-3}$ $\alpha=30$

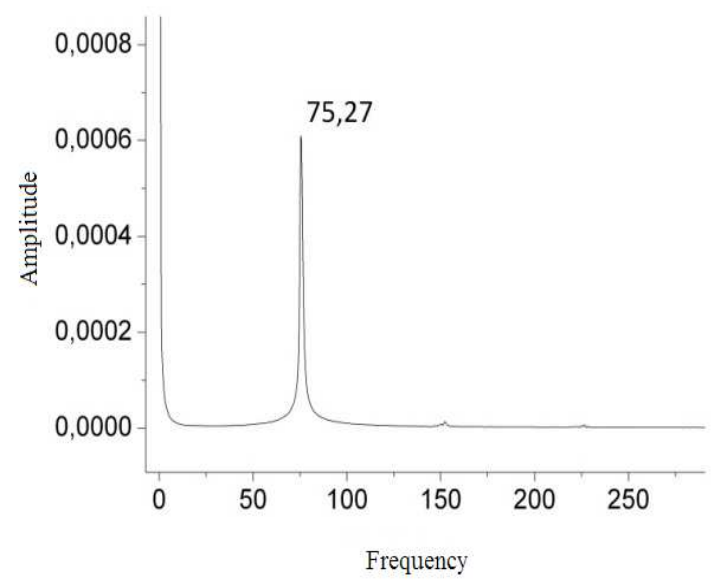

Fig. 5. Amplitude Spectrum: Unsteady solution $\mathrm{Da}=10^{-3} \alpha=30$

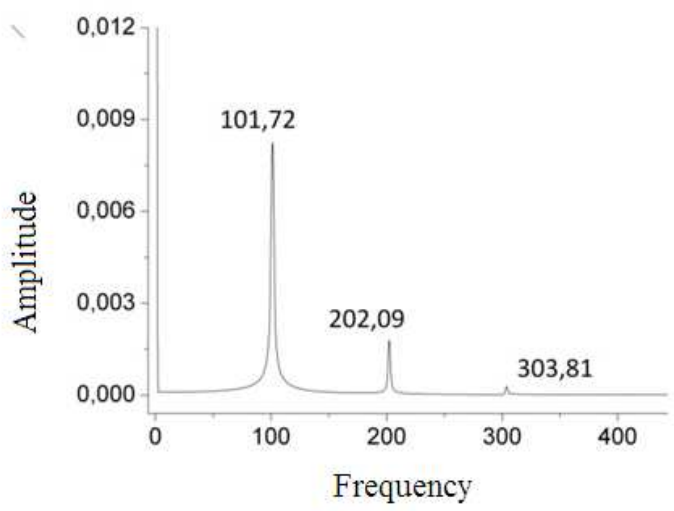

Fig. 6. Amplitude Spectrum: Cascade with harmonics.Da $=10^{-3}$ $\alpha=30$

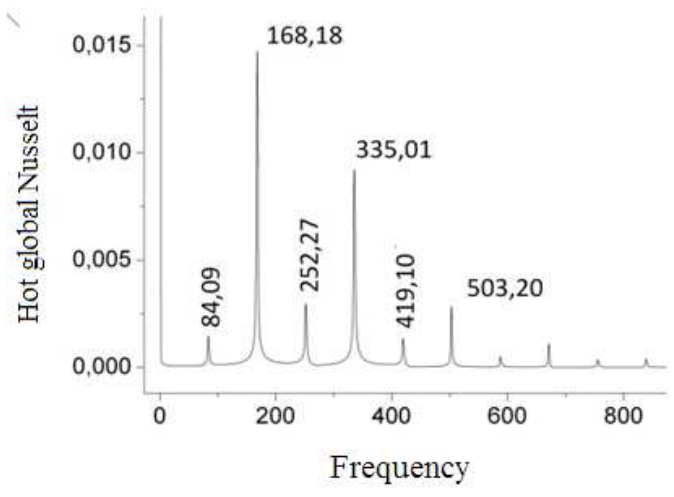

Fig. 7. Amplitude Spectrum: Cascade under harmonics $\mathrm{Da}=10^{-3} \alpha=30$

For the inclination angle equal to $60^{\circ}$, the stationary solution appears for a Rayleigh number equal to $4.8 \times 10^{5}$. The first bifurcation is highlighted for a Rayleigh number equal to $5 \times 10^{5}$ with a mesh equal to 61x61 and a smaller time step is $5 \times 10^{-6}$.The spectrum of amplitude of the Nusselt number for the periodic solution is represented in figure 8 . The chaos installs for a Rayleigh number $2 \times 10^{6}$. We note that for a given Darcy number the appearance of the stationary solution is influenced by the inclination angle of the cavity. The larger the angle is the more there is a delay. We noticed that the chaotic solution is early. The stationary solution is delayed to 60 degrees and the chaotic phenomenon appears rather. For the angle 30 the stationary solution and the chaotic solution correspond respectively to $\mathrm{Ra}=4.6 \times 10^{6}$ and $\mathrm{Ra}=2 \times 10^{7}$. We notice that for the two inclination angle values $\left(30^{\circ}\right.$ and $\left.60^{\circ}\right)$ and when the Darcy number is small, the convection does not start quickly, the stationary solution is delayed even more than the Rayleigh is large. Figures 9 and 10 show the stream function and the isotherms respectively for the two inclination angles and for Darcy number equal to $10^{-3}$. For the inclination angle equal to $30^{\circ}$ the flow structure is described by three cellular. The large cell spreads along the diagonal of the cavity favouring the heat transfer. The isothermal forms are characterizing of thermal boundary layers along the walls. We note that for the inclination angle equal to $60^{\circ}$ the flow structure is composed of one cellular.

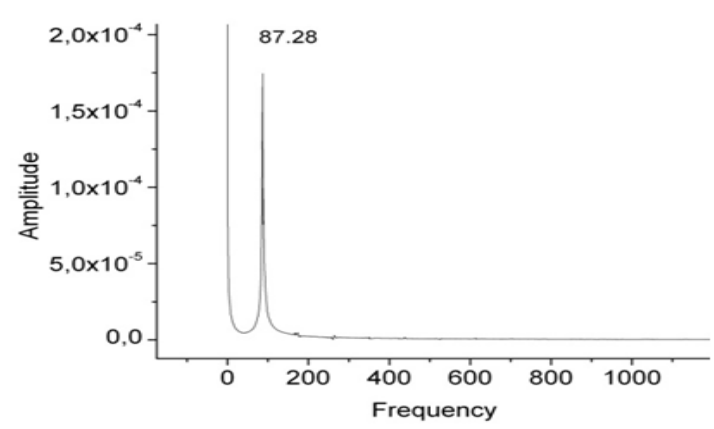

Fig. 8. Amplitude Spectrum: Unsteady solution $\mathrm{Da}=10^{-3} \alpha=60$ 


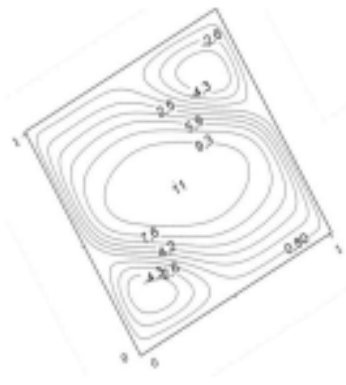

(a)

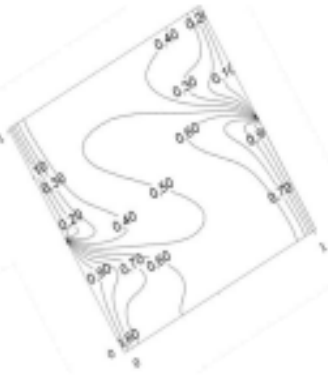

(b)
Fig. 9. a) Stream function b) isothermal $\mathrm{Da}=10^{-3} \alpha=30$

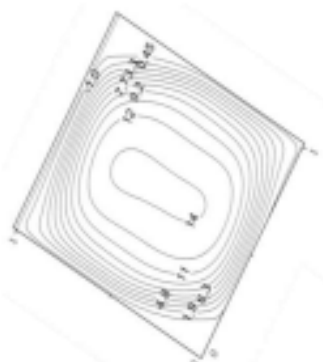

(a)

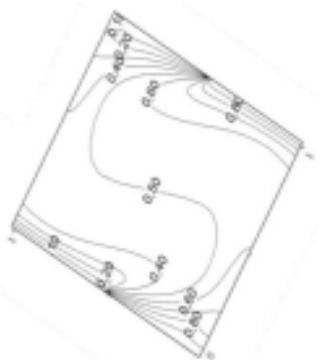

(b)
Fig. 10. a) Stream function b) Isothermal $\mathrm{Da}=10^{-3} \alpha=60$

\section{Conclusion}

In a numerical study of natural convection in air in a square cavity filled with a porous media, the roads towards chaos with the cavity inclination angle and the Darcy number was investigated. A stability study showed that the flow undergo bifurcation sequences from steady state to convective oscillatory state. The first bifurcation observed is critical. For Darcy numbers $\mathrm{Da}=$ $10^{-3}$ and $\mathrm{Da}=10^{-5}$ and a porosity equal to 0.45 , a 30 degree inclination favours convective heat transfer. The two inclinations angle values of the cavity show that the natural convection in the cavity filled with a porous medium undergoes a deterministic chaos. We have noticed that for an inclination angle equal to $30^{\circ}$, the scenario of the roads towards the chaos is in agreement with the one of Feigenbaum [13]. For the inclination angle equal to $60^{\circ}$, the scenario is that of Ruelle and Takens [14] which is by quasi periodicity.

\section{Keywords}

Natural convection, Porous media, Instability, Chaos, Attractor, Bifurcation.

\section{References}

1. M. Kaviany, Springer Verlag. (1994)

2. D. A. Nield and A.Bejan, Springer Verlag, New York (1999).
3. D.Poulikakos and A.Bejan, Int.J.Heat Mass Transfer, 26, pp. 1805-1814 (1983).

4. M. Mamou, P. Vasseur, and R. Bilgen, Int.J. Heat Mass Transfer, 38, pp.1787-1798 (2001).

5. A.A, Merrikh, and A.A Mohammed, Int.J.Heat MassTransfer, 45, pp.4305-4313 (2002).

6. T, Basak, S,.Roy, T, Paul and Pop, I, 2006, Int. J. Heat Mass Transfer, 49, pp. 1430-1441(2006).

7. J.J. Vadász, J.P. Meyer, and G.Saneshan, Transp. Porous Media, 103, pp. 279-294 (2014).

8. P. Kiran and B..S Bhadauria, Transp. Porous. Media, 107, pp.745-763 (2015).

9. B.S. Bhadauria, and P. Kiran, Int.J. Heat Mass Transfer, 84, pp. 610-624 (2015).

10. P. Vadasz, Fluids, 2, 26, doi: 10.3390/fluids 2020026 (2017).

11. G. De Vahl Davis, Int. J. Num. Meth. Fluids, 3, pp.249-264. (1983)

12. A.Ndame, Thèse Doctorat Universite de Poitiers. (1992)

13. P.Berge and Y. Pomeau, cinquième édition, Hermann. (1998).

14. D.Ruelle, and F.Takens, Com. Math.Phys,20,pp.167192 (1971) 